\title{
Article \\ Numerical Optimization of Refractive Index Sensors Based on Diffraction Gratings with High Aspect Ratio in Terahertz Range
}

\author{
Oleg Kameshkov ${ }^{1,2}$, Vasily Gerasimov ${ }^{1,2, *(D)}$ and Boris Knyazev ${ }^{1,2}$ (D) \\ 1 Budker Institute of Nuclear Physics SB RAS, 630090 Novosibirsk, Russia; o.kameshkov@g.nsu.ru (O.K.); \\ ba_knyazev@phys.nsu.ru (B.K.) \\ 2 Department of Physics, Novosibirsk State University, 630090 Novosibirsk, Russia \\ * Correspondence: v.v.gerasimov3@gmail.com
}

check for updates

Citation: Kameshkov, O.; Gerasimov, V.; Knyazev, B. Numerical Optimization of Refractive Index Sensors Based on Diffraction Gratings with High Aspect Ratio in Terahertz Range. Sensors 2022, 22, 172. https:/ / doi.org/10.3390/s22010172

Academic Editor: Dragan Indjin

Received: 13 December 2021

Accepted: 25 December 2021

Published: 28 December 2021

Publisher's Note: MDPI stays neutral with regard to jurisdictional claims in published maps and institutional affiliations.

Copyright: (C) 2021 by the authors. Licensee MDPI, Basel, Switzerland. This article is an open access article distributed under the terms and conditions of the Creative Commons Attribution (CC BY) license (https:// creativecommons.org/licenses/by/ $4.0 /)$.

\begin{abstract}
Terahertz surface plasmon resonance (SPR) sensors have been regarded as a promising technology in biomedicine due to their real-time, label-free, and ultrasensitive monitoring features. Different authors have suggested a lot of SPR sensors, including those based on 2D and 3D metamaterials, subwavelength gratings, graphene, and graphene nanotube, as well as others. However, one of the traditional approaches to realize high sensitivity SPR sensors based on metal diffraction gratings has been studied poorly in the terahertz frequency range. In this article, a linear metal rectangular diffraction grating with high aspect ratio is studied. The influence of the grating structure parameters on the sensor sensitivity is simulated. Effects arising from different ratios of depth and width were discovered and explained. The results show that the sensitivity can be increased to $2.26 \mathrm{THz} / \mathrm{RIU}$ when the refractive index range of the gas to measure is between 1 and 1.002 with the resolution $5 \times 10^{-5}$ RIU.
\end{abstract}

Keywords: terahertz range; linear diffraction gratings; surface plasmon resonance; refractive index sensing

\section{Introduction}

Fast and ultrasensitive surface plasmon resonance (SPR) sensors are a well-proven technology of refractive index and spectra substance measurement in the optical frequency range [1]. It is based on the electron surface wave excitation in the Kretschman scheme on thin metallic films. A surface plasmon can be considered as an evanescent wave propagating along the metal-dielectric interface. The electric field decays normally to the interface, providing a high sensitivity of the method to changes on the surface. The tiny spatial dimension (less than the wavelength) of surface plasmon enables characterization of objects of submicron size.

Terahertz $(\mathrm{THz})$ radiation is the part of electromagnetic radiation between the microwave and infrared regions, which lies in the frequency range of $0.3-3 \mathrm{THz}$. Terahertz waves are non-ionizing, able to pass through many dry dielectrics, and are strongly absorbed in polar solutions. Owing to the relatively large dimension of terahertz waves, they can be used for study of changes in super micron-sized objects. Terahertz radiation also tends to be very sensitive to the rotation vibration of molecules and thus makes it possible to obtain information that is not available with other analytical and imaging techniques. All this makes terahertz spectroscopy a promising tool for biological research [2-4].

High narrow peaks in the spectra and high concentration of the electromagnetic field are required for detection of low concentrations of the substance and tiny changes in the dielectric medium. The use of SPR in the terahertz frequency range on thin metal films is limited due to the high values of the optical constants of metals [5,6]. To overcome this problem, different authors suggest their solutions based on 2D and 3D metamaterials, subwavelength gratings, graphene, and graphene nanotube, as well as others [7-9]. For 
example, metagratings, which can act both as couplers and sensors, attract the increased attention of researchers [10-12]. Xiangjun Li et al. have used one of these metagratings and showed that their device achieved a high sensitivity of $335 \mathrm{GHz} / \mathrm{RIU}$ and a detection limit less than 0.0001 RIU with a frequency resolution of $33.5 \mathrm{MHz}$. [12]. Another type of the sensor design based on subwavelength gratings and Otto prism coupler was proposed by $\mathrm{Ng}$ et al. [13]. Such sensors were developed in the works of other authors [13-20], who obtained the maximum sensitivity to be as high as $3.57 \mathrm{THz} / \mathrm{RIU}$. The corresponding figure of merit (FOM) is 3966 [20]. However, one of the traditional approaches to realize high sensitivity SPR sensors based on metal diffraction gratings has been studied poorly in the terahertz frequency range.

In 2004, O'Hara et al. was the first to experimentally investigate the excitation of terahertz surface plasmon polariton (SPP) wave with a metal grating and TDS spectroscopy [21]. They determined that the coupling process was efficient, but the lifetime of SPP was very short. This is because the coupling and decoupling processes happened at the same time. Researchers suggested that another lattice profile, dielectric coating, or some other change in the system configuration, would result in a coupling/decoupling compromise. In 2008, Nazarov et al. published an article in which they optimized the excitation of terahertz SPP on structured metallic surfaces by varying grating parameters [22]. They analyzed the governing process of the coupling phenomenon and propagation of SPP over flat and corrugated structures. Other researchers investigated numerically and experimentally diffraction gratings with a trapezoidal profile [23]. They showed how to use multi-section groove gratings for efficient excitation of broadband THz SPPs. In 2011, Nazarov and Coutaz presented a review on SPRs and SPPs on metal diffraction linear grating applications and explorations, but there was no mention of sensing applications [24]. Later, Spevak et al. investigated the features of SPR excitation on small semiconductor diffraction gratings [25]. In 2020, Sathukarn et al. used the rigorous coupled-wave analysis (RCWA) method to design terahertz SPR diffraction linear rectangular gratings providing as a refractive index sensor [26]. They parametrically analyzed the gratings and found optimal parameters for surface plasmon resonance. They showed that their sensor provided a sensitivity of $500 \mathrm{GHz} / \mathrm{RIU}$ and a detected resolution of $0.01 \mathrm{RIU}$, working in the frequency interrogation.

In this paper, we investigate a one-dimensional periodic metal rectangular diffraction grating with high aspect ratio for terahertz SPR sensing. First, we introduce the configuration of the proposed terahertz SPR sensor and its simulation analogue. Next, we analyze the influence of the groove width and depth on the resonance and mode excitation of the diffraction grating. Finally, terahertz SPR sensors with different grating configurations are examined via characterization of analytes with different refractive indices for gas sensing.

\section{SPR Sensing System-Coupling Mechanism}

\subsection{Coupling of Surface Waves to a Cavity Mode}

The considered structure for terahertz SPR sensing is shown in Figure 1a. It consists of the 1D linear metal diffraction grating surrounded by the analyte. The metal grating has a rectangular profile with the period $p$, width $w$, and depth $d$. A collimated terahertz beam with TM polarization and wavelength $\lambda_{\text {inc }}$ is incident on the sensor at the specific angle $\theta$. After interacting with the analyte and the grating, the light is sent to the detector, which records the reflection spectra. To excite a SPR it is necessary to satisfy the momentum matching condition $k_{\|}=k_{s p p}$, where $k_{\|}$is the parallel part of the incident light wavevector $k_{\text {inc }}$ and $k_{s p p}$ is the surface plasmon polariton wavevector. Upon the excitation of the SPR, a dip is observed in the reflection spectrum. Since the position of the dip depends on the properties of the analyte, the coupling frequency varies with the refractive index. 


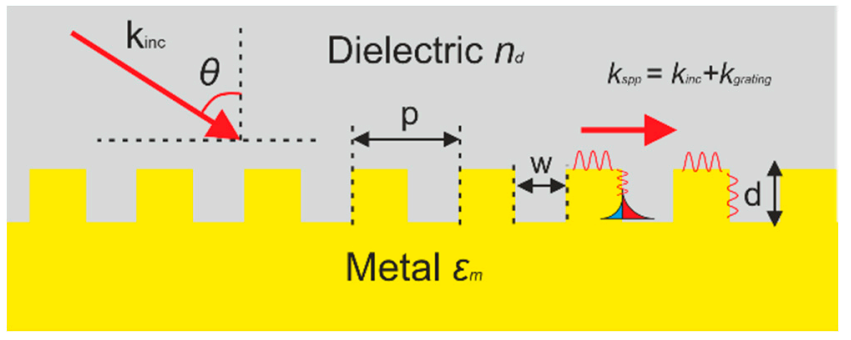

(a)

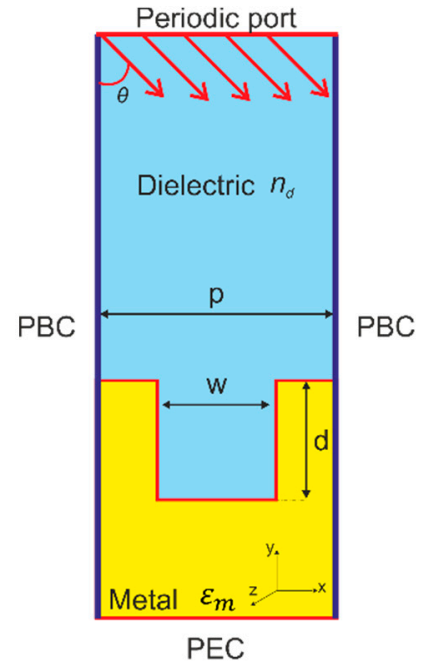

(b)

Figure 1. (a) Scheme of surface plasmon polariton resonance excitation with metal diffraction grating; (b) Numerical scheme to analyze SPR.

The surface plasmon polariton wavevector $k_{s p p}$, propagating at the interface between the metal layer with the dielectric permittivity $\varepsilon_{m}$ and the dielectric layer with the dielectric permittivity $\varepsilon_{d}$, can be written as follows [27]:

$$
k_{s p p}=\frac{2 \pi}{\lambda_{i n c}} \sqrt{\frac{\varepsilon_{m} \varepsilon_{d}}{\varepsilon_{m}+\varepsilon_{d}}} .
$$

The $k_{s p p}$ value is greater than the parallel part of the incident light wavevector $k_{\|}=$ $\frac{2 \pi}{\lambda_{i n}} n_{d} \sin \theta$. The diffraction grating acts like a coupler and adds the grating wavevector $m \frac{2 \pi}{p}$ to $k_{\|}$. Due to this, the momentum matching condition can be rewritten as

$$
n_{d} \sin \theta+m \frac{\lambda_{i n c}}{p}= \pm \sqrt{\frac{\varepsilon_{m} \varepsilon_{d}}{\varepsilon_{m}+\varepsilon_{d}}},
$$

where $m=0, \pm 1, \pm 2, \ldots$ is the diffraction order and the sign \pm on the right corresponds to the negative and positive diffraction order, respectively. In the terahertz frequency range, the dielectric permittivity of metal is high and $\varepsilon_{m} \gg \varepsilon_{d}[5,6]$, so for $m=1$ it follows from Equation (2) that

$$
p=\frac{\lambda_{\text {inc }}}{\left(1-n_{d} \sin \theta\right)}
$$

\subsection{Fabry-Perot Cavity for SPP Modes}

Another resonance that can appear when an incident wave interacts with a diffraction grating is associated with the Fabry-Perot (FB) resonance. It occurs when an excited surface wave is reflected multiple times from the sidewalls of the cavity. Consistent with a free-space Fabry-Perot resonator, the resonance condition for a resonator utilizing SPPs propagating between a pair of reflective sidewalls of the cavity is satisfied when an integer number $(s)$ of half-wavelengths fits on the length of the cavity:

$$
w=s \frac{\lambda_{s p p}}{2}
$$




\subsection{Rectangular Waveguide Cavity Resonance Mode}

In general, a deep rectangular groove is a rectangular waveguide with a cross section $w \times d$, then the critical wavelength at which the waveguide mode can exist is

$$
\lambda_{q l}=\frac{2}{\sqrt{\left(\frac{q}{w}\right)^{2}+\left(\frac{l}{d}\right)^{2}}}
$$

where $(q, l)$ are the number of half-periods of the electromagnetic oscillations along the dimension $w$ and $d$, respectively.

\section{Numerical Scheme}

The numerical data were obtained by the finite element method with the use of the Comsol Multiphysics software. We calculated the reflection of $\mathrm{THz}$ beam with frequency interrogation. The calculation scheme is shown in Figure 1b. The single groove is the unit Floquet cell with a periodic boundary condition (PBC) applied along the direction of periodicity. The excitation source is the periodic port in the boundary to inject TM-polarized terahertz radiation into the computational domain. The metal is simulated by the Drude model with the plasma frequency $\omega_{p}$ and damping frequency $\omega_{\tau}$.

The ports are used both for detection of the incident wave and for ensuring that the resulting solution leaves the model without any nonphysical reflections. For achievement of this effect, for each diffraction order $m$, its own port must be set. For calculation of the frequency spectra, the number of ports is determined with the condition $(-1-\sin \theta) \frac{p n_{d}}{\min \left[\lambda_{i n c}\right]} \leq m \leq(1-\sin \theta) \frac{p n_{d}}{\min \left[\lambda_{i n c}\right]}$. The lower part of the Floquet cell is closed with a perfect electric boundary condition (PEC). Since the penetration depth into the metal is about 50-100 $\mathrm{nm}$ in the terahertz range [27] and the metal grating thickness is much greater, this boundary condition has almost no effect.

\section{Simulation Results and Discussion}

\subsection{Optimization Process}

We developed sensors for gas analysis. Therefore, the refractive index of the analyte is assigned as $n_{d}=1$ for optimization process. The Drude parameters of copper was set $\omega_{p}=1.12 \times 10^{16} \mathrm{rad} / \mathrm{s}$ and $\omega_{t}=1.38 \times 10^{13} \mathrm{rad} / \mathrm{s}$ [28]. The incident angle and the period of the structure were chosen using Equation (3) and the condition that the number of diffraction orders will be three, including zero order. It corresponds to the maximum efficiency of SPR excitation. The period of the structure to optimize was $p=175 \mu \mathrm{m}$ and the incident angle was $\theta=15^{\circ}$, which corresponds to the resonance frequency $f_{\text {res }}=2.306 \mathrm{THz}$ $\left(\lambda_{\text {res }}=130 \mu \mathrm{m}\right)$.

The frequency reflection spectra were calculated for different widths and depths of the grooves. Next, we caught the frequency of the dip in the reflection spectra at different parameters and plotted graphs, as shown in Figure 2a. In the reflection spectrum, several dips could be observed at once when the groove depths were more than one wavelength. Each dip corresponded to its own excited mode of grating. The small points on the graph correspond to the dip maxima in the spectrum for a given groove depth and width.

Surface plasmon polaritons at the interface of the grating surface and air appear if Equation (2) is satisfied. The excitation frequency may be lower due to the influence of higher diffraction orders and interference effects. The dip position in the frequency axis in the spectrum shifts with increases in the groove depth (Figure 2a). SPPs penetrate into the grooves along their sides and interact with the fundamental mode of the groove, which leads to the appearance of a high-intensity electric field inside the grooves. 


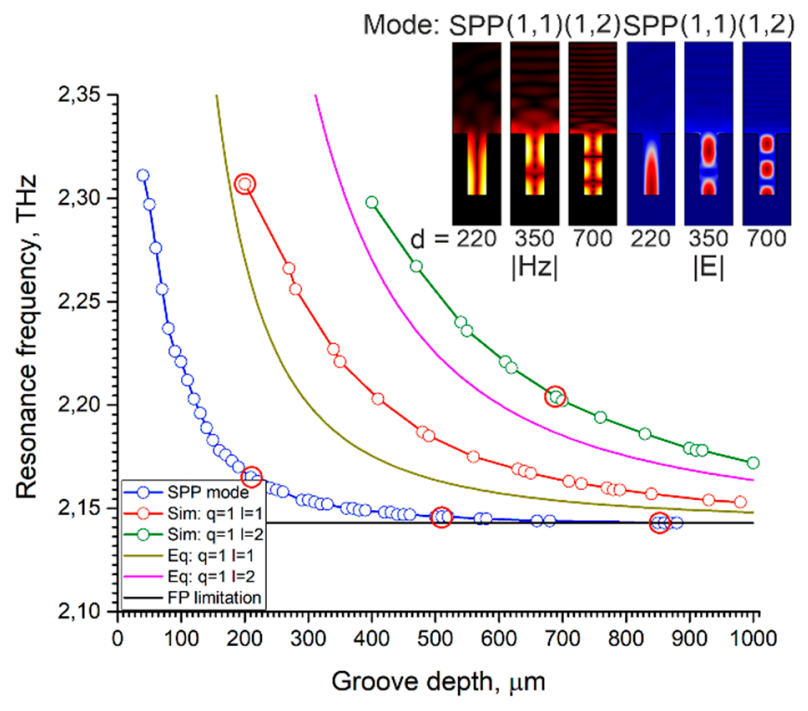

(a)

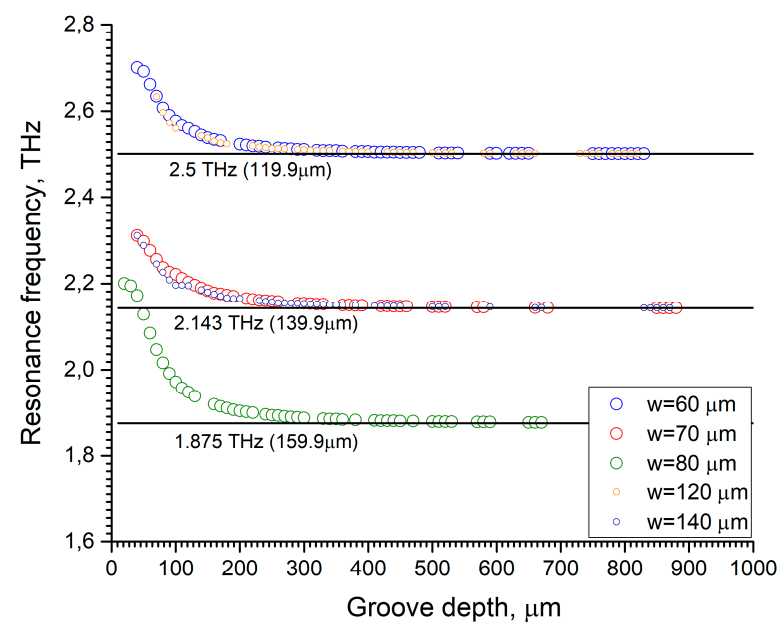

(b)

Figure 2. Results of calculation presented for copper diffraction gratings with rectangular profile and $p=175 \mu \mathrm{m}$. Incident angle of TM polarized wave $\theta=15^{\circ}$. Small points on graph plots correspond to maximum dips in spectrum for given groove depth and width. (a) Resonance frequency vs. groove depth for different modes. Width of grooves $w=70 \mu \mathrm{m}$. The solid lines correspond to FP resonance condition (black lines), $(1,1)$ mode (swamp line), and $(1,2)$ mode (pink line). Big red points correspond to grating parameters chosen for further analysis; (b) Resonance frequency vs. groove depth for SPP mode at different groove widths.

The groove width affects the Fabry-Perot resonance condition (see Equation (4)) which entails changes in the resonant response of the grating. When the groove width is much less than the wavelength, the grating can be considered as a kind of medium with subwavelength cavities, and we did not observe deep dips in the reflection spectrum. If the groove width is $\approx \lambda_{\text {res }} / 2$, resonance effects begin to appear, and modes associated with both plasmons and the Fabry-Perot effect are excited. If the groove depth is greater than the wavelength, then the grating grooves form a Fabry-Perot cavity in which plasmons interact with each other. The resonance frequency of this cavity limits the frequency of the excited plasmon in the entire system in accordance with Equation (4). The dependence of 
the frequency on the groove depth is repeated for multiple groove widths, as shown in Figure $2 b$.

In the general case, the grating groove is a rectangular resonator with a cross section $w \times d$, and then the minimum frequency at which the mode exists can be determined from Equation (5). In calculations, we observed the excitation of resonator modes, and in some cases these resonances dominate as shown in Figure 2, and the dips in the reflection spectra were deeper for a non-SPP mode.

\subsection{Sensor Sensitivity—Frequency Domain}

The main parameters that enable comparison of the sensors are the figure of merit $F O M_{f}$ and the sensitivity $S_{f}$. For the frequency spectrum, $S_{f}=d f / d n$ and $F O M_{f}=$ $S_{f} / F W H M$, where $d n$ is the refractive index change and FWHM is the full width at half maximum of the dip in the reflection spectrum of the frequency region. To obtain the $S_{f}$ and $F O M_{f}$ parameters, we varied the refractive index, as shown in Figure 3a. Each dip corresponds to its own frequency and refractive index, and therefore it is possible to plot the dependences $f_{\text {res }}\left(n_{d}\right)$ and approximate them with $f_{\text {res }}=f_{\text {offset }}+S \cdot n_{d}$ (Figure $3 \mathrm{~b}$ ).

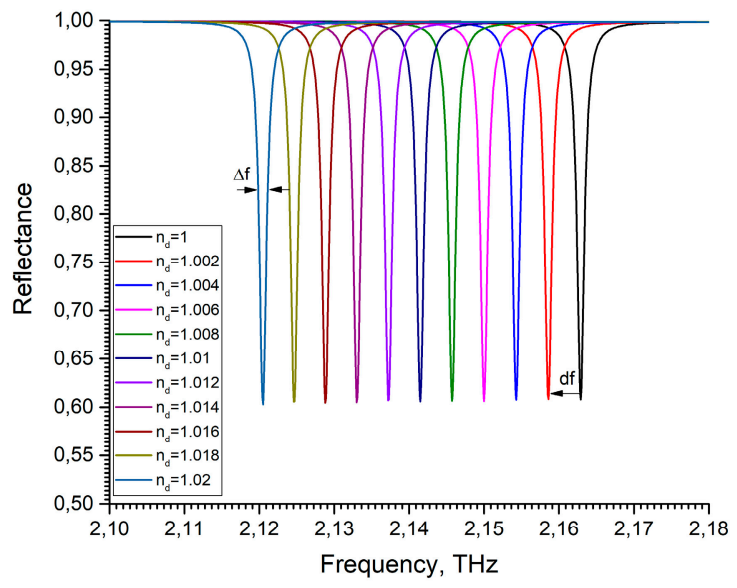

(a)

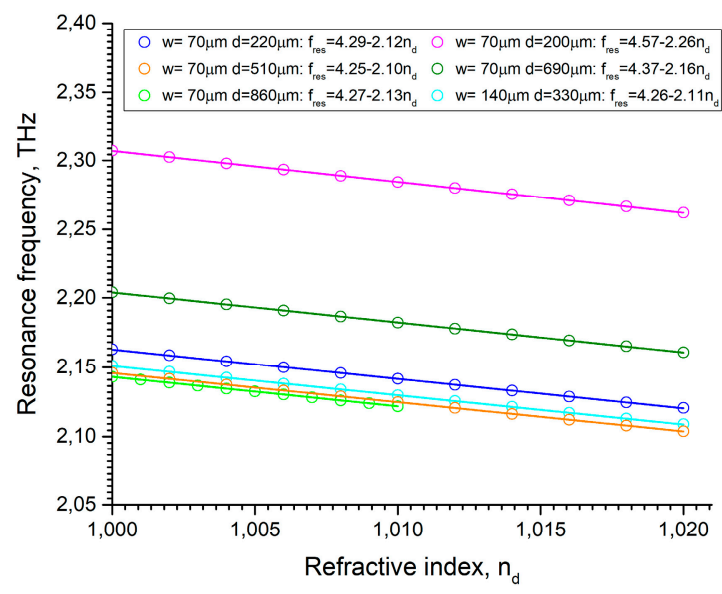

(b)

Figure 3. (a) Reflection curves of gratings filled with different refractive index analytes at $p=175 \mu \mathrm{m}$, $w=70 \mu \mathrm{m}$, and $d=220 \mu \mathrm{m} ;(\mathbf{b})$ Resonance frequencies $n_{d}$ vs. filling refractive index for different diffraction gratings. Solid lines are linear fittings.

We can see (Figure 3b) that the resonance frequency dependences of the fundamental and higher modes remain linear with respect to the refractive index. The sensitivity of the 
diffraction gratings to a change in the refractive index is almost independent on the excited mode. The maximum sensitivity that we achieved in our calculations is $2.26 \mathrm{THz} / \mathrm{RIU}$ at $p=175 \mu \mathrm{m}, w=70 \mu \mathrm{m}$, and $d=200 \mu \mathrm{m}$. The FWHM of the dips varies slightly with a change in the refractive index, and for an estimate it is sufficient to take the FWHM of the initial dip. The FWHM decreases with the increasing groove depth. The maximum FOM reached $212501 /$ RIU at $p=175 \mu \mathrm{m}, w=70 \mu \mathrm{m}$, and $d=860 \mu \mathrm{m}$.

The detection limit of the sensor is defined as

$$
\delta n_{\mathrm{d}}=\frac{\delta f_{\text {res }}}{S}
$$

where $\delta f_{\text {res }}$ is the frequency resolution defined by the resonance FWHM or the spectral resolution of the radiation source. In conventional terahertz time-domain systems, $\delta f_{\text {res }} \approx 5 \mathrm{GHz}$ can be decreased to the limit of about $100 \mathrm{MHz}$, which is comparable with the best FWHM values obtained in our simulations for the deep gratings (see Table 1). The estimation according to Equation (6) gives the refractive resolution $\delta n_{\mathrm{d}} \approx 5 \times 10^{-5}$ RIU, which has the same order as in the best optical fiber refractometers in the visible and near infrared ranges [29], and as in the ultrasensitive dielectric metagratings in the subTHz domain [12]. Besides, the obtained refraction resolution is much higher than in the most sensitive THz subwavelength metal gratings using Otto prism coupling [20].

Table 1. Parameters of diffraction gratings and their sensing possibilities.

\begin{tabular}{|c|c|c|c|c|c|c|c|c|}
\hline $\begin{array}{c}p \\
\mu \mathrm{m}\end{array}$ & $\begin{array}{c}w \\
\mu \mathrm{m}\end{array}$ & $\begin{array}{c}d \\
\mu \mathrm{m}\end{array}$ & $\begin{array}{l}\boldsymbol{\theta} \\
\circ\end{array}$ & $\begin{array}{l}f_{\text {res }} \\
\text { THz }\end{array}$ & $R$ & $\begin{array}{c}F W H M, \\
\text { THz }\end{array}$ & $\begin{array}{c}S_{f}, \\
\text { THz/RIU }\end{array}$ & $\mathrm{FOM}_{f}, 1 / \mathrm{RIU}$ \\
\hline \multicolumn{9}{|c|}{ SPP mode } \\
\hline 175 & 70 & 220 & 15 & 2.163 & 0.621 & 0.00115 & 2.12 & 1843 \\
\hline 175 & 70 & 510 & 15 & 2.146 & 0.617 & 0.0002 & 2.10 & 10,500 \\
\hline 175 & 70 & 860 & 15 & 2.143 & 0.477 & 0.0001 & 2.13 & 21,250 \\
\hline 175 & 70 & 200 & 15 & 2.307 & 0.83 & 0.00119 & 2.26 & 1899 \\
\hline 175 & 70 & 690 & 15 & 2.204 & 0.768 & 0.001 & 2.16 & 2160 \\
\hline \multicolumn{9}{|c|}{ SPP mode } \\
\hline 175 & 140 & 330 & 15 & 2.151 & 0.671 & 0.0004 & 2.11 & 5275 \\
\hline
\end{tabular}

\subsection{Sample Fabrication Method-Producibility}

Diffraction gratings in the terahertz range are made by cutting grooves in a substrate using a CNC machine or a laser, compressing molding of micro-powder and 3D printing [30-34]. Each of these methods has its own limitations. Before using one of them, it is necessary to understand under what conditions our sensor will not be in the resonance state. To estimate it, we took one grating configuration and calculated deviation of its parameters from optimal state. As can be seen from Figure 4, the grating has the following manufacturing restrictions: $p=175 \pm 13 \mu \mathrm{m}, w=70 \pm 1.5 \mu \mathrm{m}, d=220 \pm 5 \mu \mathrm{m}$. Manufacturing precision can be reached with well-developed femtosecond laser cutting machines [35], which are used in the industrial applications. 


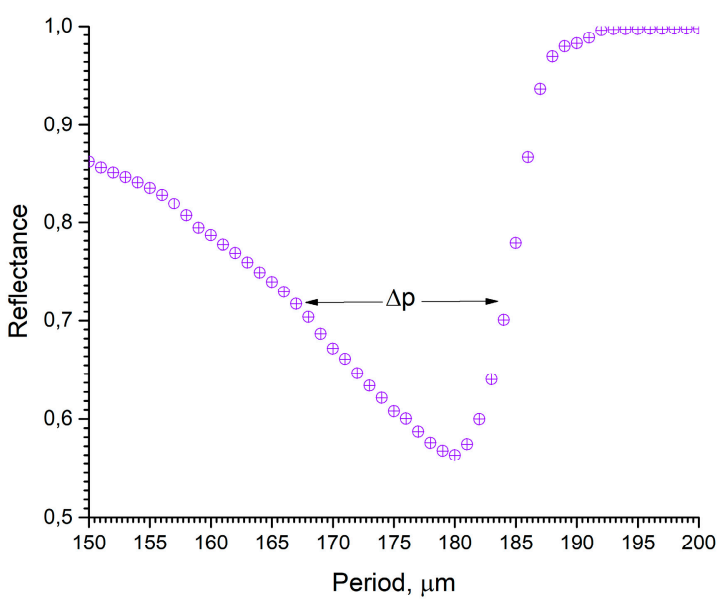

(a)

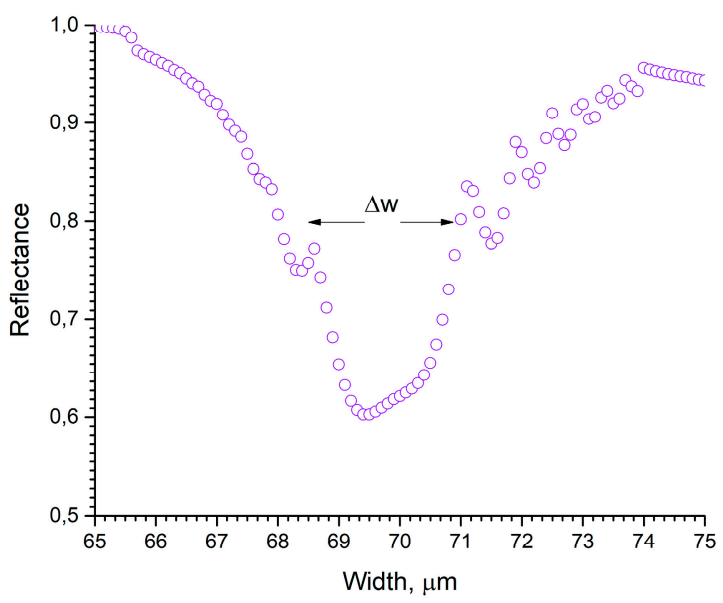

(b)

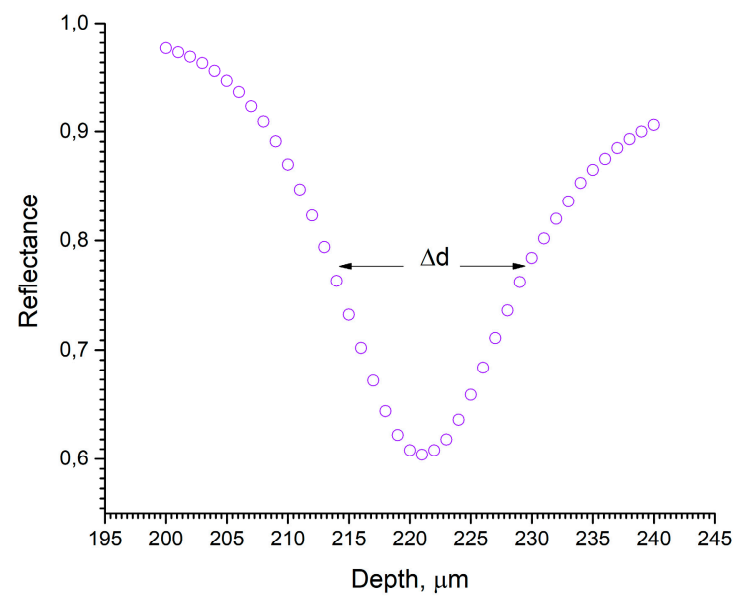

(c)

Figure 4. Assessment of manufacturing accuracy of grating parameters such as (a) period, (b) width, and (c) depth. 


\section{Conclusions}

In this study, we consider a parameter design technique for a one-dimensional periodic rectangular metallic grating with high aspect ratio for terahertz sensing. The influence of different structure parameters on the sensing sensitivity is analyzed with the use of a numerical simulation method. The sensing sensitivity can be increased to $2.26 \mathrm{THz} / \mathrm{RIU}$ via optimization of the structure parameters when the refractive index range of the gas to measure is in the range of 1 to 1.002 with the resolution $5 \times 10^{-5}$ RIU. In addition, we consider the influence of different grating modes on the sensitivity. The sensitivity of the diffraction gratings to a change in the refractive index is almost independent on the excited mode.

Author Contributions: This work was realized through the collaboration of all authors. O.K.: contributing to the main results and simulation; V.G.: reviewing and editing, scientific discussion; B.K.: editing, supervision, funding acquisition. All authors have read and agreed to the published version of the manuscript.

Funding: This research received no external funding.

Institutional Review Board Statement: Not applicable.

Informed Consent Statement: Not applicable.

Data Availability Statement: Not applicable.

Acknowledgments: The work was done for experiments at the shared research center SSTRC on the basis of the Novosibirsk FEL complex at BINP SB RAS.

Conflicts of Interest: The authors declare no conflict of interest.

\section{References}

1. Barnes, W.L.; Dereux, A.; Ebbesen, T.W. Surface Plasmon Subwavelength Optics. Nature 2003, 424, 824-830. [CrossRef]

2. Yang, X.; Zhao, X.; Yang, K.; Liu, Y.; Liu, Y.; Fu, W.; Luo, Y. Biomedical Applications of Terahertz Spectroscopy and Imaging. Trends Biotechnol. 2016, 34, 810-824. [CrossRef] [PubMed]

3. Smolyanskaya, O.; Chernomyrdin, N.; Konovko, A.; Zaytsev, K.; Ozheredov, I.; Cherkasova, O.; Nazarov, M.; Guillet, J.-P.; Kozlov, S.; Kistenev, Y.; et al. Terahertz biophotonics as a tool for studies of dielectric and spectral properties of biological tissues and liquids. Prog. Quantum Electron. 2018, 62, 1-77. [CrossRef]

4. Zaytsev, K.I.; Dolganova, I.N.; Chernomyrdin, N.V.; Katyba, G.M.; Gavdush, A.A.; Cherkasova, O.P.; Komandin, G.A.; Shchedrina, M.A.; Khodan, A.N.; Ponomarev, D.S.; et al. The progress and perspectives of terahertz technology for diagnosis of neoplasms: A review. J. Opt. 2020, 22, 013001. [CrossRef]

5. Pandey, S.; Gupta, B.; Chanana, A.; Nahata, A. Non-Drude like Behaviour of Metals in the Terahertz Spectral Range. Adv. Phys. X 2016, 1, 176-193. [CrossRef]

6. Bulgakova, V.V.; Gerasimov, V.V.; Goldenberg, B.G.; Lemzyakov, A.G.; Malkin, A.M. Study of Terahertz Spoof Surface Plasmons on Subwavelength Gratings with Dielectric Substance in Grooves. Procedia Eng. 2017, 201, 14-23. [CrossRef]

7. Gao, Z.; Wu, L.; Gao, F.; Luo, Y.; Zhang, B. Spoof Plasmonics: From Metamaterial Concept to Topological Description. Adv. Mater. 2018, 30, 1706683. [CrossRef] [PubMed]

8. Beruete, M.; Jáuregui-López, I. Terahertz Sensing Based on Metasurfaces. Adv. Optical Mater. 2020, 8, 1900721. [CrossRef]

9. Gerasimov, V.V.; Hafizov, R.R.; Kuznetsov, S.A.; Lazorskiy, P.A. Exploiting Localized Surface Plasmon Resonances in Subwavelength Spiral Disks for THz Thin Film Sensing. Appl. Sci. 2020, 10, 3595. [CrossRef]

10. Liu, Z.; Li, X.; Yin, J.; Hong, Z. Asymmetric All Silicon Micro-Antenna Array for High Angle Beam Bending in Terahertz Band. IEEE Photonics J. 2019, 11, 1-9. [CrossRef]

11. Li, X.; Liu, Z.; Yan, D.; Li, J.; Li, J.; Qiu, G.; Hou, X.; Cheng, G. Experimental Demonstration of 3D Printed Terahertz PolarizationInsensitive Flat Devices Based on Low-Index Meta-Gratings. J. Phys. D Appl. Phys. 2020, 53, 505301. [CrossRef]

12. Li, X.; Wang, L.; Cheng, G.; Hou, X.; Yan, D.; Qiu, G.; Guo, S.; Zhou, W.; Li, J. Terahertz Spoof Surface Plasmon Sensing Based on Dielectric Metagrating Coupling. APL Mater. 2021, 9, 051118. [CrossRef]

13. Ng, B.; Wu, J.; Hanham, S.M.; Fernández-Domínguez, A.I.; Klein, N.; Liew, Y.F.; Breese, M.B.H.; Hong, M.; Maier, S.A. Spoof Plasmon Surfaces: A Novel Platform for THz Sensing. Adv. Opt. Mater. 2013, 1, 543-548. [CrossRef]

14. Yao, H.; Zhong, S. High-Mode Spoof SPP of Periodic Metal Grooves for Ultra-Sensitive Terahertz Sensing. Opt. Express 2014, 22, 25149. [CrossRef]

15. Yao, H.; Zhong, S.; Tu, W. Performance Analysis of Higher Mode Spoof Surface Plasmon Polariton for Terahertz Sensing. J. Appl. Phys. 2015, 117, 133104. [CrossRef] 
16. Zhang, Y.; Hong, Z.; Han, Z. Spoof Plasmon Resonance with 1D Periodic Grooves for Terahertz Refractive Index Sensing. Opt. Commun. 2015, 340, 102-106. [CrossRef]

17. Chen, L.; Yin, H.; Chen, L.; Zhu, Y. Ultra-Sensitive Fluid Fill Height Sensing Based on Spoof Surface Plasmon Polaritons. J. Electromagn. Waves Appl. 2018, 32, 471-482. [CrossRef]

18. Chen, X.; Xiao, H.; Lu, G.; Zhao, R. Refractive Index Sensing Based on Terahertz Spoof Surface Plasmon Polariton Structure. J. Phys. Conf. Ser. 2020, 1617, 012008. [CrossRef]

19. Huang, Y.; Zhong, S.; Shi, T.; Shen, Y.; Cui, D. Terahertz Plasmonic Phase-Jump Manipulator for Liquid Sensing. Nanophotonics 2020, 9, 3011-3021. [CrossRef]

20. Zhao, R.; Lu, G.; Yin, H.; Liang, J.; Zeng, D.; Xiao, H. Terahertz Sensor Study Based on Spoof Surface Plasmon Polaritons. Int. J. Antennas Propag. 2020, 2020, 2504626. [CrossRef]

21. O’Hara, J.F.; Averitt, R.D.; Taylor, A.J. Terahertz Surface Plasmon Polariton Coupling on Metallic Gratings. Opt. Express 2004, 12, 6397. [CrossRef] [PubMed]

22. Nazarov, M.; Garet, F.; Armand, D.; Shkurinov, A.; Coutaz, J.-L. Surface Plasmon THz Waves on Gratings. Comptes Rendus Physique 2008, 9, 232-247. [CrossRef]

23. Martl, M.; Darmo, J.; Unterrainer, K.; Gornik, E. Excitation of Terahertz Surface Plasmon Polaritons on Etched Groove Gratings. J. Opt. Soc. Am. B 2009, 26, 554. [CrossRef]

24. Nazarov, M.; Coutaz, J.-L. Terahertz Surface Waves Propagating on Metals with Sub-Wavelength Structure and Grating Reliefs. J. Infrared Milli. Terahz. Waves 2011, 32, 1054-1073. [CrossRef]

25. Spevak, I.S.; Kuzmenko, A.A.; Tymchenko, M.; Gavrikov, V.K.; Shulga, V.M.; Feng, J.; Sun, H.B.; Kamenev, Y.E.; Kats, A.V. Surface Plasmon-Polariton Resonance at Diffraction of THz Radiation on Semiconductor Gratings. Low Temp. Phys. 2016, 42, 698-702. [CrossRef]

26. Sathukarn, A.; Jia yi, C.; Boonruang, S.; Horprathum, M.; Tantiwanichapan, K.; Prasertsuk, K.; Thanapirom, C.; Kusolthossakul, W.; Kasamsook, K. The Simulation of a Surface Plasmon Resonance Metallic Grating for Maximizing THz Sensitivity in Refractive Index Sensor Application. Int. J. Opt. 2020, 2020, 3138725. [CrossRef]

27. Maier, S.A. Plasmonics: Fundamentals and Applications; Springer: Berlin/Heidelberg, Germany, 2007; Volume 1.

28. Ordal, M.A.; Bell, R.J.; Alexander, R.W.; Long, L.L.; Querry, M.R. Optical Properties of Fourteen Metals in the Infrared and Far Infrared: $\mathrm{Al}, \mathrm{Co}, \mathrm{Cu}, \mathrm{Au}, \mathrm{Fe}, \mathrm{Pb}, \mathrm{Mo}, \mathrm{Ni}, \mathrm{Pd}, \mathrm{Pt}, \mathrm{Ag}, \mathrm{Ti}, \mathrm{V}$, and W. Appl. Opt. 1985, 24, 4493. [CrossRef]

29. Urrutia, A.; Villar, I.D.; Zubiate, P.; Zamarreco, C.R. A Comprehensive Review of Optical Fiber Refractometers: Toward a Standard Comparative Criterion. Laser Photonics Rev. 2019, 13, 1900094. [CrossRef]

30. Scherger, B.; Born, N.; Jansen, C.; Schumann, S.; Koch, M.; Wiesauer, K. Compression Molded Terahertz Transmission BlazeGrating. IEEE Trans. Terahertz Sci. Technol. 2012, 2, 556-561. [CrossRef]

31. Busch, S.F.; Born, N.; Koch, M.; Fischer, B. Terahertz Reflection Gratings Made by Room-Temperature High-Pressure Molding. J. Infrared Milli. Terahz. Waves 2013, 34, 413-415. [CrossRef]

32. Squires, A.D.; Constable, E.; Lewis, R.A. 3D Printed Terahertz Diffraction Gratings and Lenses. J. Infrared Milli. Terahz. Waves 2015, 36, 72-80. [CrossRef]

33. Busch, S.F.; Weidenbach, M.; Balzer, J.C.; Koch, M. THz Optics 3D Printed with TOPAS. J. Infrared Milli. Terahz. Waves 2016, 37, 303-307. [CrossRef]

34. Ornik, J.; Zhang, Y.; Schneider, M.; Taherkhani, M.; Alaboz, H.; Koch, M. THz Gratings Produced by Laser Cutting. In Proceedings of the 2019 44th International Conference on Infrared, Millimeter, and Terahertz Waves (IRMMW-THz), Paris, France, 1-6 September 2019; IEEE: Paris, France, 2019; pp. 1-2.

35. Linas, J.; Dovilè, M.; Gabrielius, K.; Vytautas, P. Femtosecond lasers: The ultimate tool for high-precision 3D manufacturing. Adv. Opt. Technol. 2019, 8, 241-251. [CrossRef] 